\title{
Localisation Challenges in Postmenopausal Hyperandrogenism
}

Seong K Cheah ${ }^{1}$, Ahmad Miremadi ${ }^{2}$, Sidrah Khan ${ }^{1}$, Anitha Mathews ${ }^{1}$, Singhan M Krishnan ${ }^{1}$.

${ }_{1}^{1}$ Department of Endocrinology and Diabetes, Hinchingbrooke Hospital, North West Anglia Foundation Trust, UK

${ }^{2}$ Department of Pathology, Addenbrooke Hospital, Cambridge University Hospital Trust, UK

\section{CASE HISTORY AND WORKUP}

A 48 years old lady with BMI of $46 \mathrm{~kg} / \mathrm{m}^{2}$ was postmenopausal since age 45. Due to abdominal discomfort she had an abdominal CT, which incidentally identified bilateral adrenal adenoma $(9 \mathrm{~mm}$ on right, $18 \mathrm{~mm}$ on left, with fat content), Fig 1.

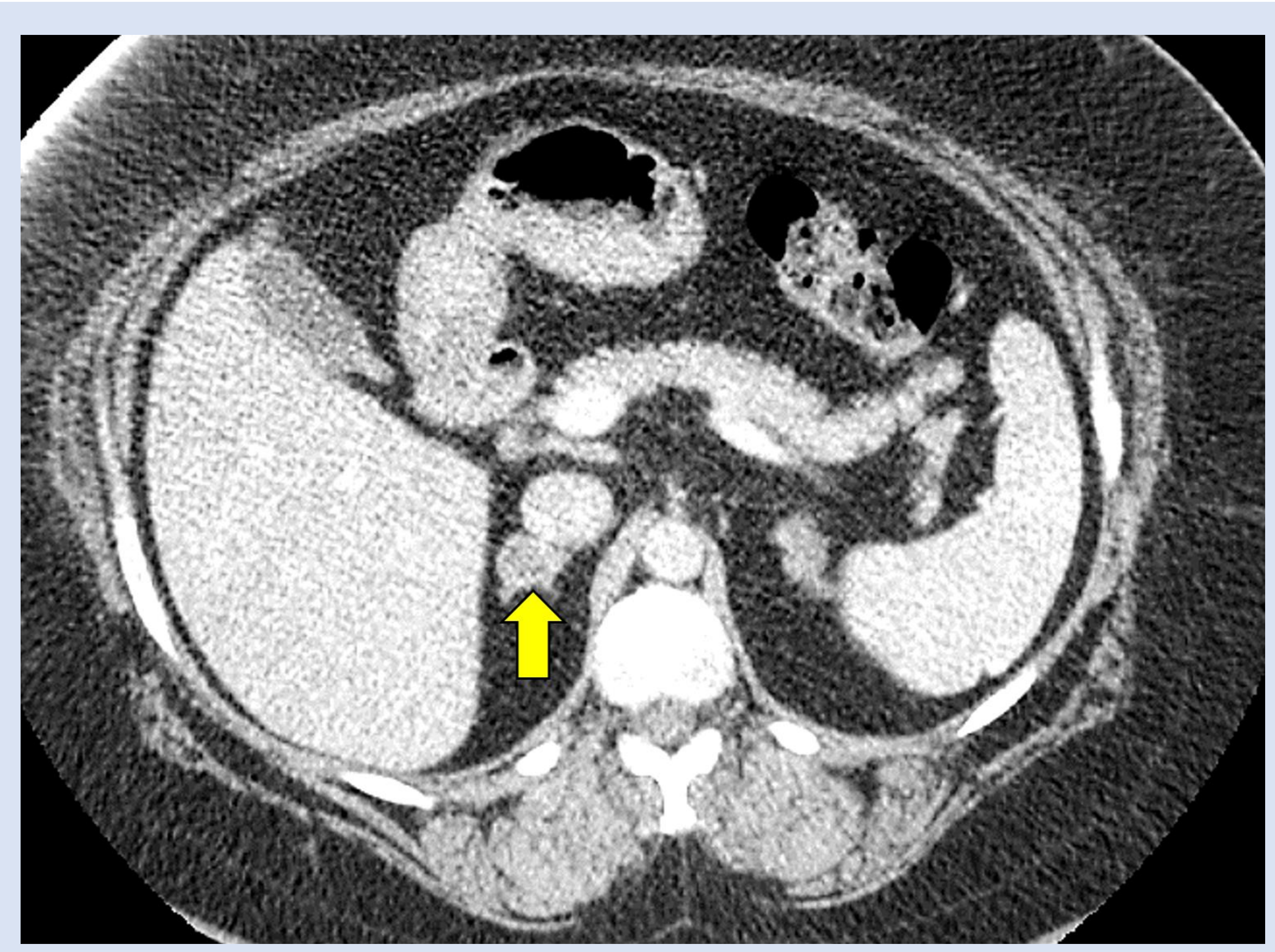

Fig 1. CT scan showing adrenal adenoma (yellow arrow)

This resulted in Endocrinology referral and a history of gradually worsening hirsuitism was uncovered.

Her hyperandrogenism was confirmed biochemically with markedly elevated testosterone at $6.5 \mathrm{nmol} / \mathrm{L}(0.0-1.8)$, leading to a search for adrenal and ovarian source.

The preliminary endocrinological blood investigations were normal.

- Adrenal androgens were normal:

- Androstenedione $3.3 \mathrm{nmol} / \mathrm{L}(0.9-4.8)$

- DHEAS $0.9 \mu \mathrm{mol} / \mathrm{L}(0.7-7.8)$

- 17-OH progesterone $3.4 \mathrm{nmol} / \mathrm{L}(0.0-5.0)$

$\mathrm{FSH}$ and $\mathrm{LH}$ were of post-menopausal levels.

TFT, ACTH, 9am Cortisol, prolactin and CA125 were normal.

Her body habitus limited ultrasound imaging and hence MRI abdomen and pelvis was performed.

Again, adrenal was identified to be adenomatous as suggested by signal-drop. Interestingly, the ovaries are reported to have normal appearance with small follicles.

A delineation between adrenal and ovarian aetiology was unclear at this stage while patient preference and body habitus limited the option for specific venous sampling.

An overnight dexamethasone suppression test had led to cortisol suppression to $38 \mathrm{nmol} / \mathrm{L}$ excluding Cushing's syndrome, while testosterone remained non-suppressed at $5.8 \mathrm{nmol} / \mathrm{L}$, suggesting an ovarian androgen source.

Following this, a trial of GnRH analogue (subcutaneous Leuprorelin $3.75 \mathrm{mg}$ monthly) had led to suppression and normalisation of testosterone $(0.6 \mathrm{nmol} / \mathrm{L})$ after 2 months, consistent with ovarian hyperandrogenism.

However, she found GnRH analogue intolerable due to flushing, precluding its adoption as long term therapeutic measure. Laparoscopic bilateral oophorectomy then resulted in persistent normalisation of post-op testosterone level $(0.6 \mathrm{nmol} / \mathrm{L}$ at $2 \mathrm{month})$, further affirming the ovarian source.

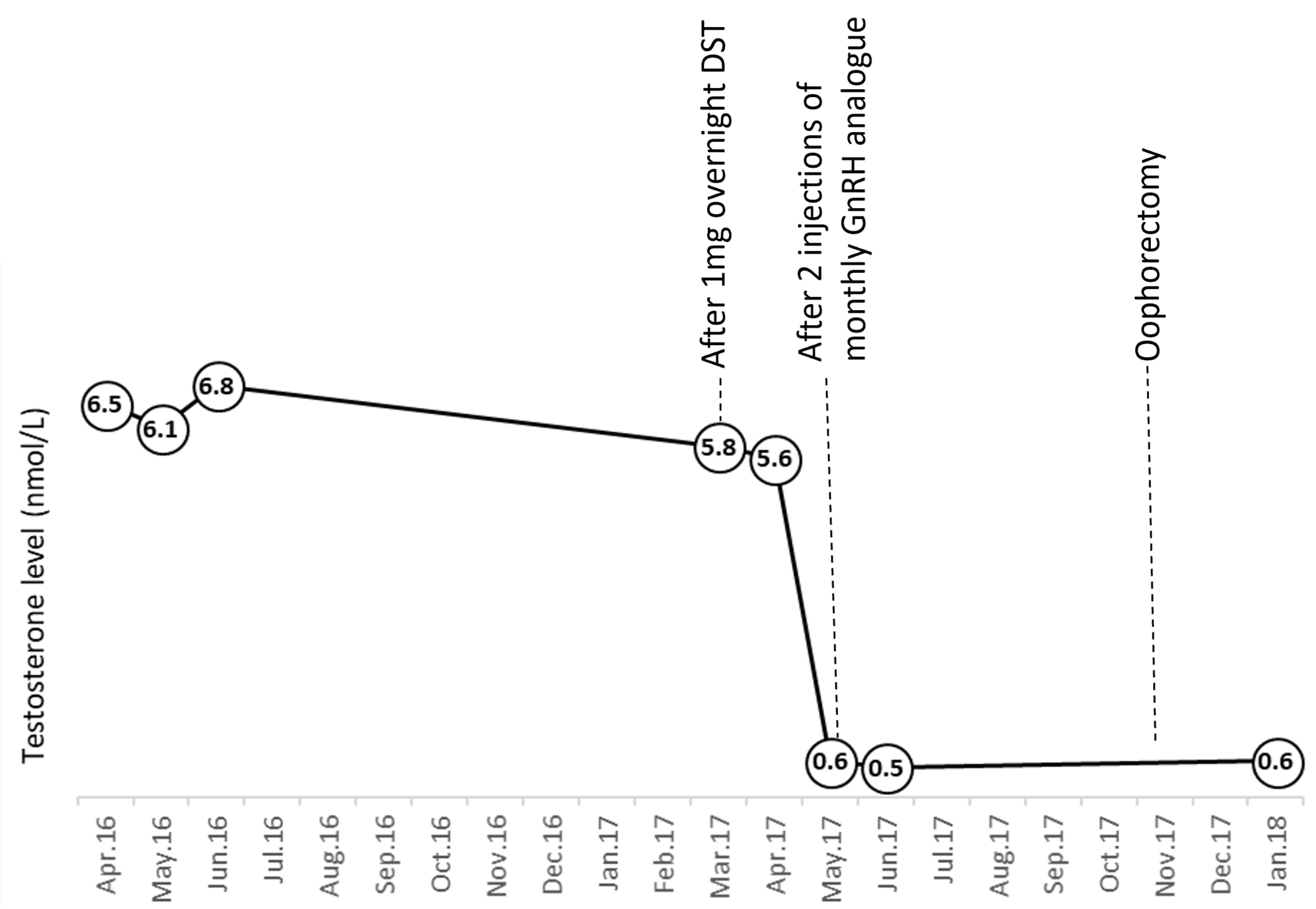

Fig 2. Testosterone level over time in response to investigatory or surgical events

Contrary to the MRI findings, her ovaries were found to be significantly large (17 and $23 \mathrm{~cm}^{3}$ ) for a postmenopausal lady, who typically has mean ovarian volume of $1.3-3.7 \mathrm{~cm}^{3}$. The pathological findings of inhibinpositive luteinised cells in ovaries confirmed hyperthecosis (Fig. 3 and Fig 4)

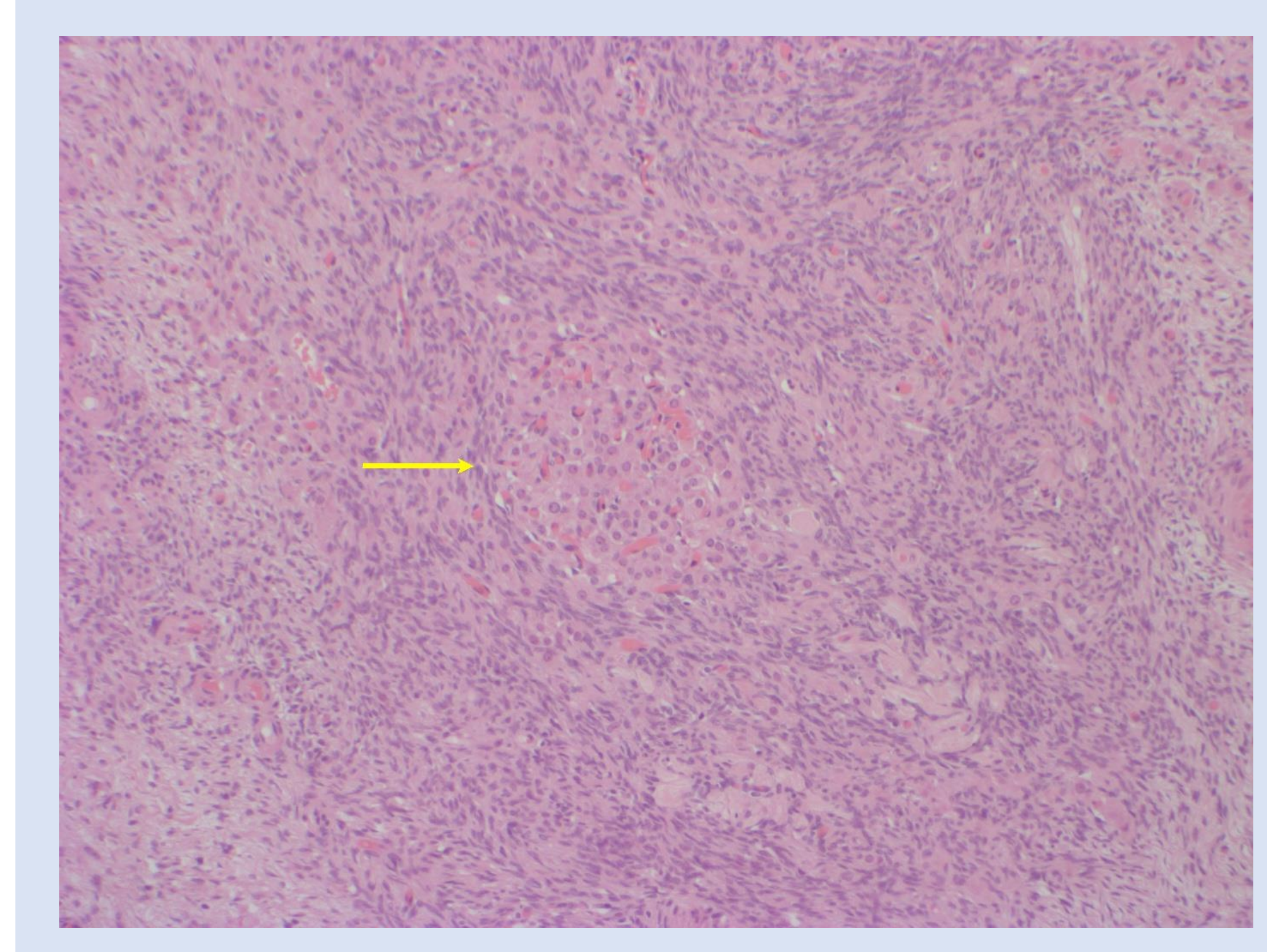

Fig 3. Ovaries: showing a nest of luteinised stromal cells (yellow arrow, $x 20$ objective).

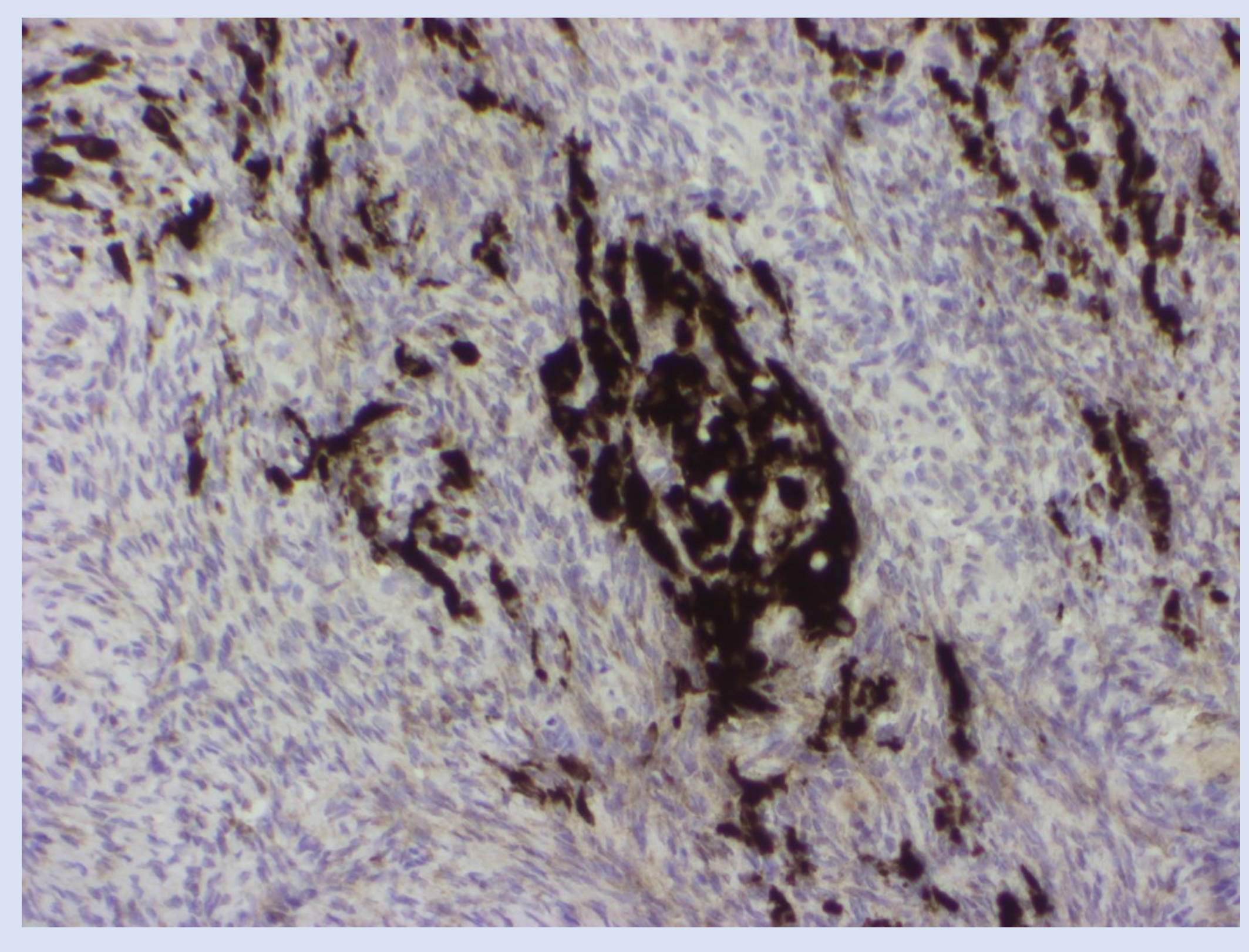

Fig 4. Ovaries: inhibin-positive luteinised cells as single cells and in clusters

\section{DISCUSSION}

Localising postmenopausal hyperandrogenism can be challenging. Markedly elevated level of testosterone $>5 \mathrm{nmol} / \mathrm{L}$ may represent malignant aetiology, and warrants localisation of its adrenal or ovarian source. Specific venous sampling may be limited by its relative invasiveness, availability and patient's body habitus or preference. Less invasive tests will prove to be helpful in this context.

As illustrated in this case, adrenal aetiology may be supported by concomitant rise of DHEAS and suppressible testosterone by overnight dexamethasone test. Whereas GnRH analogue may suppress testosterone of ovarian source, a response which may subsequent justify definitive oophorectomy.

Society for Endocrinology

a world-leading authority on hormones

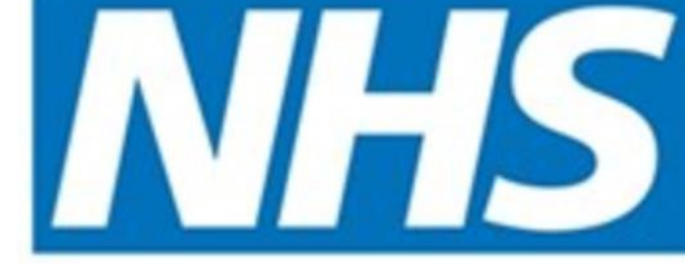

North West Anglia NHS Foundation Trust 